\title{
Adaptations and Advancement of Biologic Immunotherapy in the Management of Immunologically Cold Solid Malignancies
}

\author{
Authors: \\ *Aaron C. Shang, ${ }^{1,2}$ Kristen E. Galow ${ }^{2}$ \\ 1. University of Oxford, Medical Sciences Division, Oxford, UK \\ 2. Hackensack Meridian School of Medicine at Seton Hall University, Nutley, New \\ Jersey, USA \\ *Correspondence to aaron.shang@kellogg.ox.ac.uk
}

Disclosure: $\quad$ The authors have declared no conflicts of interest.

Received: $\quad 18.12 .19$

Accepted: $\quad 29.01 .20$

Keywords: $\quad$ Cancer, checkpoint inhibitor, chemotherapy, epigenetic, immunogenic, immunotherapy, medicine, oncolytic, radiotherapy, tumour, vaccine.

Citation: $\quad$ EMJ Oncol. 2020;DOI/10.33590/emjoncol/19-00206

\begin{abstract}
Contemporary breakthroughs within cancer immunotherapy are frequently cited amongst the most promising of therapeutic directions for medical oncology and perioperative solid tumour management. However to date, the efficacy of treatment of immunologically derived therapeutic modalities is limited to a few highly selective malignancies, exemplified by leukaemia or renal cell carcinoma. Many solid tumours exhibiting low immune activity, i.e., immunologically 'cold', such as highly aggressive pancreatic cancers, have correspondingly become regarded as inappropriate for prospective immunotherapeutic modulation. Standard approach in these tumours therefore relies upon early-stage identification and curative surgical resection, an identifiably imperfect option in both progression temporality and deterrence of metastatic disease.
\end{abstract}

Fundamentally predicated upon the therapeutic activation of existing systemic immune resources, selectively towards malignant transformed cellular subpopulations, current cancer immunotherapy heavily utilises monoclonal antibody checkpoint inhibitors (i.e., PD-1, PDL-L1, CTLA-4) influencing resultant upregulation of physiologic immune activation pathways. These correspondingly enhance immunologic function and interfere with carcinogenesis. With ongoing development in the scientific understanding of complex tumour microenvironment interactions and subclonal heterogeneity, increasingly promising investigations have developed. These include the effective management of low immune activity cold solid tumours with original immunogenic cofactor therapies as well as immune modulation in conjunction with co-operative chemotherapeutic, radiological, or surgical intervention.

Advancements in novel combination immunotherapies as well as innovative downstream management courses offer great optimism for the applicability of emerging cancer immunotherapy to prospective treatment of cold tumours. This review comprehensively analyses and discusses notable current research directions in the field and underscores future directions for continued scientific progress alongside relevant clinical applications. 


\section{INTRODUCTION}

Increased awareness has emerged regarding the critical role of immunotherapy within translationalcancermanagementand personalised medicine. Encompassing notable developments such as widely adaptable monoclonal immune checkpoint antibodies, donor immune cell transfusion, and direct cytokine incorporation have brought about significant advancements in effective noninvasive clinical management opportunities for a spectrum of cancers. Fundamental to the majority of current cancer immunotherapy modalities is the adaptation of pre-existing host immune resources to selectively detect and eliminate carcinogenesis, resulting in enhanced T-lymphocyte infiltration (TIL) of solid tumour tissues." This cascading process induces malignant cell apoptosis and parent tumour necrosis, accompanied by marked reduction in aggressive invasion as well as metastatic behaviours in vivo. ${ }^{2}$ Therefore, modern cancer immunotherapy heavily predicates upon clinical modification of cancer cells' erroneous 'elimination, equilibrium, escape' lifecycle components wherein growth transitions uncontrollably from physiologic to pathologic patterns through avoidance of natural immunologic growth-inhibiting mechanisms. Under healthy conditions, several immune checkpoints, PD-1, PD-L1, and CTLA-4, downregulate immune responses to prevent autoimmunity and systemic exhaustion. Relevant to the development of cancer treatments, immune checkpoints are of value for therapeutic targeting in the form of inhibitory antibodies (immune checkpoint inhibitors [ICI], such as ipilimumab, anti-CTLA-4, pembrolizumab, anti-PD-1, durvalumab, and anti-PD-L1), as induced checkpoint interferences allow for expanded antagonism against cancer cell functions (Figure 1). ${ }^{3-5}$

Whereas patient-centred immunotherapy outcomes have been demonstrated for many haematologic malignancies and immune-active 'hot' solid tumours including lung and breast cancer subtypes, ${ }^{6}$ counterpart cold tumours have concurrently become progressively recognised for poor response to immunologically based therapy. This is exemplified via intrinsically poor TIL infiltration as well as being immunologically ignorant and therefore expressing lower levels of relevant checkpoint receptors or even targetable biomarkers including major histocompatibility complex class 1.7 However, cold tumours demonstrate alternative populations of less therapeutically utilised immune infiltration by myeloid progenitors. $^{8}$ A significant and contentious debate revolves around whether cold immunologic tumours, such as primary pancreatic and prostate cancers, may prove to be realistic targets for cancer immunotherapy. This review provides a comprehensive discussion of current literature into such cold tumour immunotherapy and evaluates its applicability, perceived limitations, and future directions.

\section{MODERN MANAGEMENT AND EMERGING MODALITIES}

As a foundation for point-of-care therapeutic guidelines, distinction between hot and cold tumours relies on the Immunoscore ${ }^{\circledR}$ classification, a robust and standardised system based on T-cell $(\mathrm{CD} 3+/ \mathrm{CD} 8+)$ prevalence at the centre of the tumour and exterior invasive margin. ${ }^{9}$ It is scored from IO-14, with IO indicating low infiltration at both measured locales and therefore characterising cold tumours. Immunoscore has demonstrated to be more accurate than pathologic tumour staging as well as clinical differentiation status, nuclear atypia, or lymphovascular invasion severity at predicting both patient prognosis and immunotherapeutic response. ${ }^{10}$ By convention, IO-11 are considered cold tumours, 12 neutral, and 13-14 immunologically hot. Furthermore, marked decreases in immunotherapy effectiveness within solid tumour treatment correspondingly occur below the 12 tumour Immunoscore threshold.11-13 Importantly, this scale does not differentiate between causes that may result in differing levels of tumoural TIL infiltration such as fundamental deficiency of tumour-associated antigens, defective antigen-presenting cell recruitment, or substandard T-lymphocyte costimulation with activation upon antigen presentation. ${ }^{14}$ Furthermore, intra-Immunoscore (i.e., 12) differences in longitudinal outcome and tumour behaviour by geographical T-cell distribution remain under evaluation. ${ }^{15,16}$ 

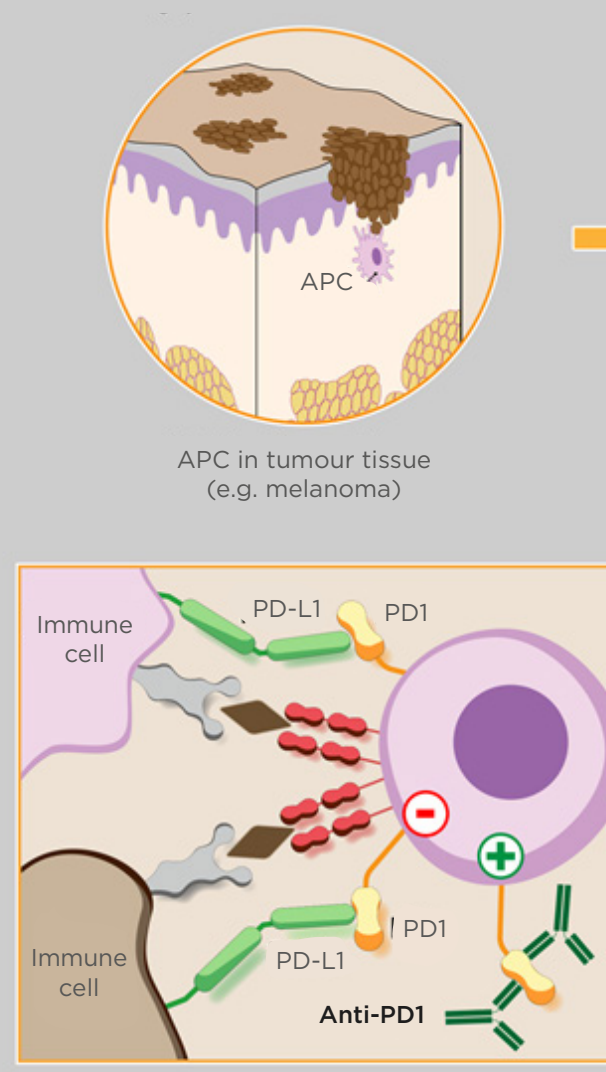

Tumour microenvironment

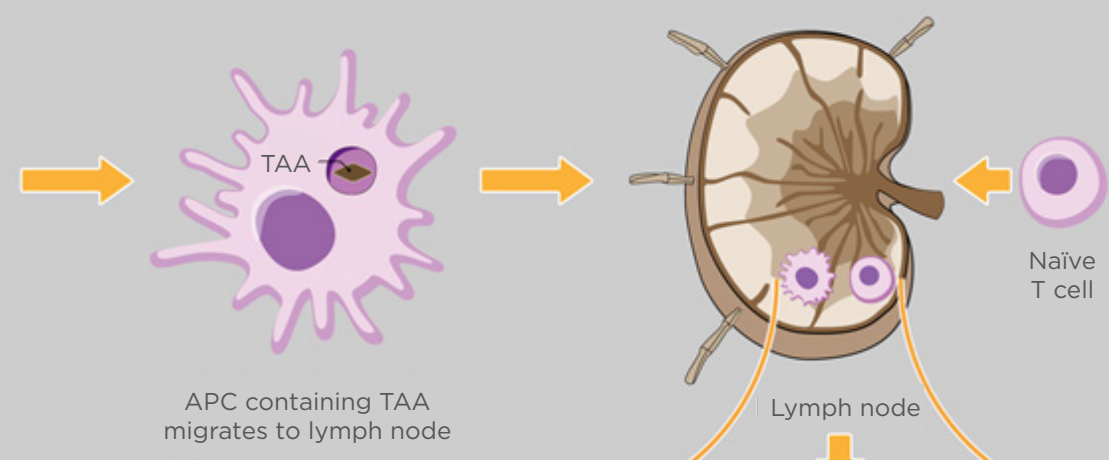

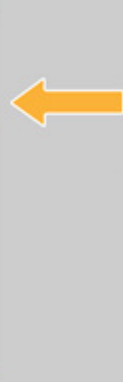

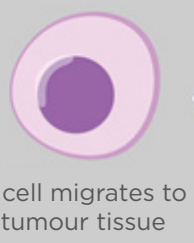

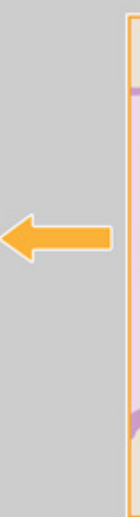

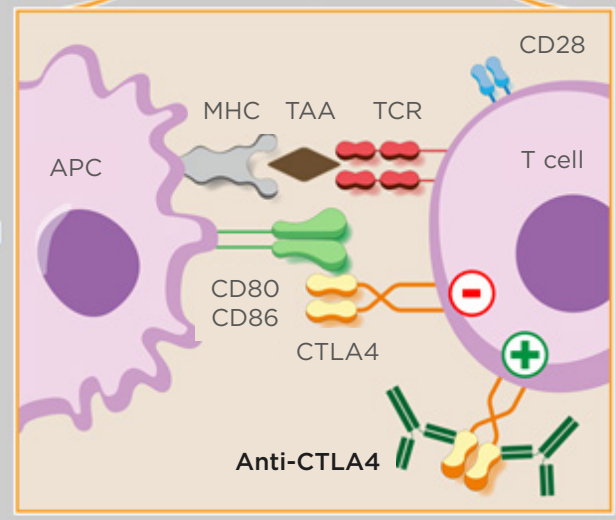

T-cell priming

Figure 1: Model of cancer therapy by inhibition of negative immune regulation (CTLA-4, PD-1).

Cancer immunology by downregulation of physiologic immune checkpoints (i.e., CTLA-4, PD-1) therapeutically enhance host immune system responses to malignant tumorous cellular growth. Implementation of CTLA-4/PD-1 inhibitors improve activation of T cells against tailorable tumour-associated antigens which consequently encourages carcinogenic apoptosis alongside reduced neoplasia.

APC: antigen-presenting cell; MHC: major histocompatibility complex; TAA: tumour-associated antigen; TCR: T-cell receptor.

Adapted from www.hegasy.de. ${ }^{3}$

In vivo efficacy of cancer immunotherapies relies heavily upon host immune system adaptation as well as the sustained presence of baseline anticarcinogenic immunology. Factors which increase immune function (i.e., pro-TIL inflammatory modulators or high infiltration) or immune knowledge of tumour markers therefore generally improve treatment response, whereas corresponding deficiencies (i.e., immunosuppression or low TIL-populated microenvironments) produce inhibited effects of treatment. Classification of cold tumours by Immunoscore (IO-11) has been shown to strongly correlate with poor clinical outcomes and predictably reduced patient response to immunologically-derived treatments (both $p<0.001)^{1718}$ Interest in immune-nonresponsive solid tumour management has therefore focussed upon the introduction of targetable factors or stimuli into a fundamentally silent immunologic landscape, which may in turn establish foundations upon which immunomodulation may be efficaciously introduced ${ }^{19-21}$ (Figure 2$)^{22}$ 


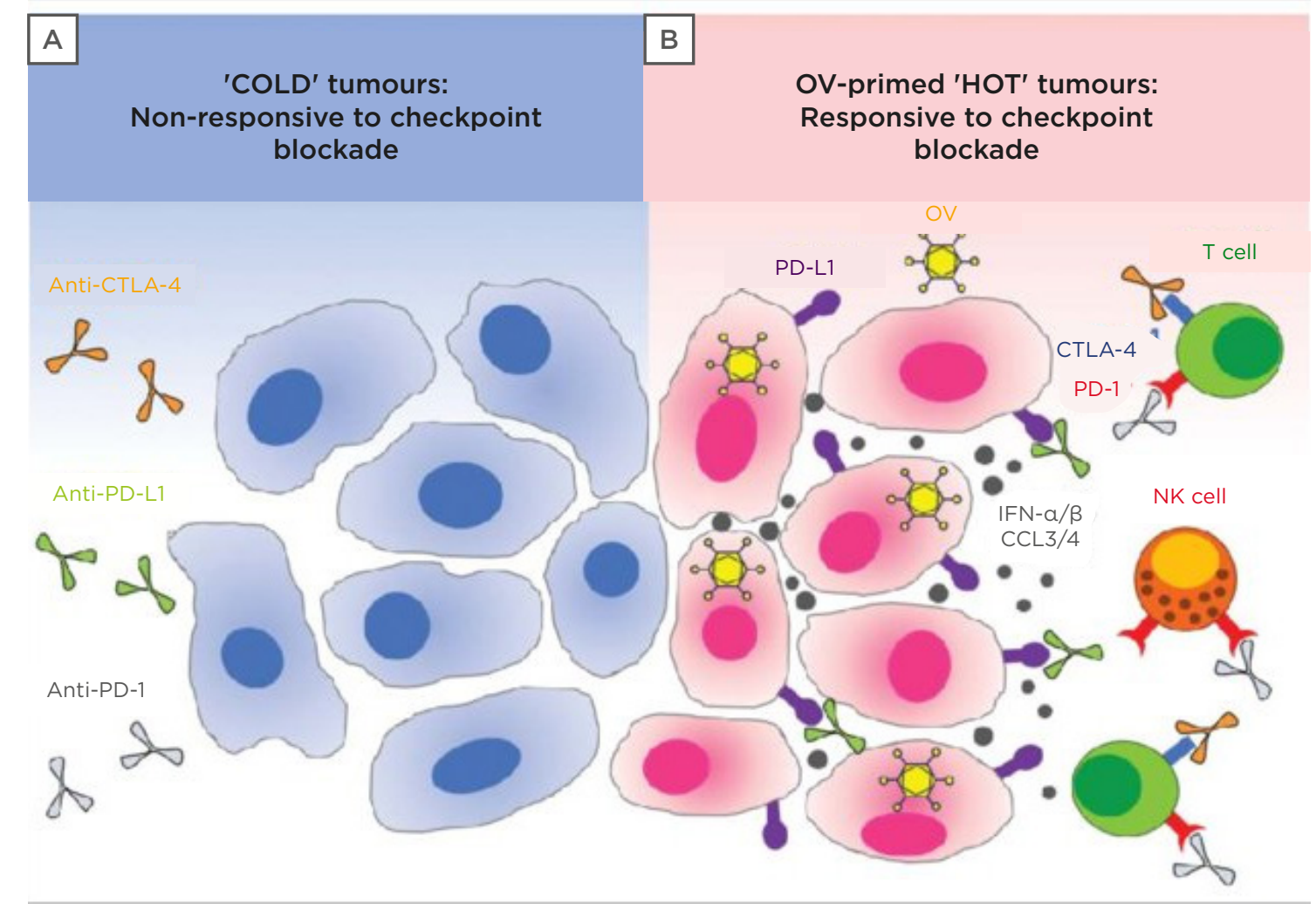

Figure 2: Therapeutic potentiality of cold tumour immune response by oncolytic virology.

'Cold' solid cancer tumour cells exhibit low immunologic targetability and corresponding responsiveness to immunotherapeutic modulation given low cell-surface protein expression (A). Clinical implementation of oncolytic viruses (B) may modulate local and systemic tumour cell behaviours, including enhanced immune receptor availability, leading to positive immunotherapy applicability.

IFN: interferon; NK: natural killer; OV: oncotherapeutic viruses.

Adapted from Gujar et al, 2018.22

These treatment approaches are categorised into the following overarching means of immune facilitation and provocation: combination chemotherapy, radiotherapy, immunostimulation or direct oncolytic virotherapy, and the novel peptide-based cancer 'vaccines'.

\section{Combination Chemotherapy and Immunotherapy}

Clinical inquiry into methods of cooperative, dual chemo- and immunotherapy to stimulate cold solid tumour immunogenicity represents a prominent and longstanding direction of interest. Trials in this subsection of pharmacotherapy occur predominantly between expansion of recognisable tumour cell adjuvanticity and antigenicity. Literature suggests that high mutational burden and intratumoural heterogeneity project poor immunotherapy prognoses 23,24 and evolving data informs utility of artificial 'neo-antigens' which may be presented through direct genotoxic chemotherapeutic courses (i.e., cyclophosphamide) and reliably produce downstreamchangesinimmune relevancethrough increased tumoural antigenicity. ${ }^{25}$ By broadly inserting drug-induced precursor DNA lesions to neoplastic cell populations, physiologic cascades including the well-researched CGAS-STINGIP3 pathway are hypothesised to upregulate local tumour immunogenicity, allowing formerly cold tumours to be more efficaciously targeted by present standardised immunomodulatory pathways. ${ }^{26}$ Ongoing trials ${ }^{27-29}$ introducing direct pharmaceutical cGAS-STING-IP3 hyperactivity (investigative drug ADU-S100) alongside immunotherapy (pembrolizumab) are similarly recipients of interest attributable to preliminary 
data suggesting high efficacy against diverse cold lymphomas, primary otolaryngologic lesions, and secondary metastatic lesions. ${ }^{30}$

Noted limitations across animal models with concerted efforts to singularly increase tumoural antigenicity via neo-antigens revolve around unpredictable levels of induced neo-antigen epitope expression, which given commonly moderate-to-low prevalence correlate to modest effectiveness. ${ }^{31}$ This presentation derives from clinical reality that many DNA-damaging chemotherapeutic agents act upon mature cancer cells, which leads to only mitotic daughter cells receiving an introduced mutation for immune generation. Mutation rates for chemotherapeutics aimed at increasing antigenicity within established solid tumours are therefore low in comparison to the initial replication processes of the same cancer. This is because uncontrolled growth would likely have gone through hundreds of rounds of replication by the time of drug-influenced DNA damage. ${ }^{32}$ Overall neoantigen efficacy and prevalence, regardless of chemotherapeutic toxicity and dosage, by result inherently remain less appreciable than that of baseline hot solid tumours in regard to the aforementioned challenges in clonal proliferation. An additional challenge is that mature tumours also contain a significant proportion of non-dividing, fully mature cancer cells. Chemotherapeutic genotoxicity in these tumoural components would essentially produce no immunogenic benefit and, given their abundance, many chemotherapeutic induction courses for immune treatment remain considerably limited in effect size and consistency. Nonetheless, unpredictable antigenicity improvements from numerous chemotherapeutic agents may concurrently influence adjuvanticity, the secondary activation of apoptotic or tumour necrosis pathways, through exocytosis of damageassociation molecular patterns. Simultaneously, concentrated local apoptosis of tumour cells may induce a systemic IFN-a immune response, resulting in heightened recruitment of $\mathrm{CD} 4+$ / CD8+ memory and cytotoxic T cells. A replicated pre-clinical finding in murine models ${ }^{25,33}$ has instigated considerable interest in the practicality of commonly used anticancer anthracyclines, taxanes, or oxaliplatin among others as immuneinstigating co-therapy. ${ }^{34}$

\section{Epigenetic Medicine}

Alongside traditional cancer pharmaceuticals, the application of fledgling epigenetic agents toward upregulation of cold tumour antigenicity as well as presentation pathways has in vitro shown to enhance the penetrance of therapeutically utilisable immunogenic markers. Through demethylation of silenced antigen codons common to tumours clonally selected for immune resistance, DNA methyltransferase inhibitor drugs ( i.e., decitabine) have been reported in literature to introduce open transcription frames correlative to subsequent tumour production of highly immune active and targetable peptides. $^{35}$ Additionally, a majority of currently available epigenetic drugs (DNA methyltransferase inhibitors and histone-lysine $\quad \mathrm{N}$-methyltransferase $\mathrm{EZH} 2$ inhibitors) are well-replicated as being able to significantly reduce silencing of intratumoural TH1-response cytokines, a process that heavily regulates $\mathrm{CD} 8+\mathrm{T}$ cell infiltration associated with impaired patient prognoses. ${ }^{36}$ Therefore, without inducing detectable chemotherapeutic damage into host systems or tumour cells directly, epigenetic drugs and demethylating agents at present appear to nonetheless carry significant promise in cold cancer immunotherapy supplementation. Currently, multiple early stage clinical trials are examining the safety profile and pharmacodynamics for numerous proposed epigenetic-inclusive combination regimens. ${ }^{37,38}$ Prominent trials include the EMERGE trial ${ }^{39}$ (Phase II) for gastrointestinal cancers, investigating anti-PD-L1 immunotherapy with domatinostat, a novel class 1-selective histone deacetylase inhibitor hypothesised to increase cold gastrointestinal tumours' immunogenicity and successive susceptibility to follow-up checkpoint inhibition. ${ }^{40}$

\section{Radiation Oncology and Direct Stimulatory Immunotherapy}

Perhaps a more direct procedure for inducing immunogenicity suitable for immunotherapeutic targeting lies in radiotherapy that utilises ionising radiation directed at controlled tumour cell immunogenic cell death. Via elaborated mechanisms within which temporal homogeneity of tumour cell apoptosis may expand toward systemic anticarcinogenic benefit, ${ }^{41}$ immunogenic radiotherapy coupled with $\mathrm{ICl}$ is currently 
regarded with optimism for control of varied cancers. These include trials ranging from systemic metastatic disease to treatment of resistant cold neoplasms. ${ }^{42,43}$ Through highdose radiation of aggressive cold malignancy such as pancreatic-head or non-small-cell lung carcinoma, ${ }^{44,45}$ integrative radio- and immune- combined therapy in animal models have exhibited the capacity to cause systemic immune upregulation more systemically than localised chemotherapy precursors. ${ }^{46}$ In practise, this indirect systemic augmentation suggests the capacity to concomitantly protect against localisable primary metastases as well as reduce the severity of secondary malignancies both known and yet undiscovered. Precise mechanisms of reliably generalised immune protective effects remain under investigation, although some recent studies suggest that exaggerated posttreatment increases cold tumour-specific CD103+/CD141+ murine and human protein, quantities which are relatively less scarce in these CD3+/CD4+/CD8+ immune-ignorant tumoural populations. ${ }^{35}$

Emerging work into the dynamic interplay of tumour microenvironments with systemically administered tailorable immunotherapies has yielded encouraging findings. Through combination regimens of localised immune response stimulatory agents (i.e., inactivated viral vaccine), an early-phase clinical trial which co-administered anti-PD-1 antibody (pembrolizumab) has reported productive utility for the transformation of low immune activity neoplasms into more targetable levels of immune infiltration and drug modifiable expression. ${ }^{47,48}$ Contemporary investigation demonstrating strong consistency and reliability of these preliminary findings have lent further value to this particular line of research. ${ }^{45}$ However, to date there remains the need for prospective Phase III trials to assess macroscopic interrelationships and rare adverse effects between locally stimulating viral administration alongside concurrent immune intervention. Additional clinical clarification in key areas yet undetermined and inherent to the described treatment combinations include whether patient demographics, disease stage or determinable genotypic (mutational degree) variation, and medical history with potential comorbidities may influence prognosis from these dual treatment courses. ${ }^{49}$

\section{Anticarcinogenic Virotherapy and Neoadjuvant 'Vaccination'}

Outside of immune response-provoking effects for viral vaccine introduction into the cold tumour microenvironment, interdisciplinary investigations delineate that engineered oncolytic viruses may play a role in the efficacious immune elimination of mutated tumour cells. ${ }^{50}$ Whereas the direct resultant effect of oncolytic viruses often involves cytotoxicity and highly specific cancer cell death locally comparable to chemotherapy and radiotherapy, literature has suggested more stable and prolonged systemic immunogenicity from viral oncolysis. This is through mechanisms including but not limited to ICD instigation, DAMP exocytosis, and viral activation of antigen-presenting cells (major histocompatibility complex Class II) all of which combine to upregulate host immune functionality ${ }^{51}$ (Figure 3). ${ }^{52}$ Infected cancer cells within immunologically cold tumours, which frequently confer considerable challenges for antibody checkpoint inhibitors or other traditional immunotherapy methods, have also been shown to be more reliably targeted by antitumour antigen-specific T-lymphocyte driven immune reactions in animal models. This is hypothesised as a result of systemic immune recognition resources being able to recognise oncolytic viruses, if not markers on infected host tumour cells themselves. Mediated elimination of both components consequently has demonstrated desirable tumour-reducing outcomes in preliminary research. ${ }^{53}$ More recently, advanced melanoma and squamous metastatic disease management trials ${ }^{54,55}$ utilising oncolytic viruses alongside pembrolizumab (PD-1 inhibitor) and talimogene laherparepvec (a melanomaand sarcoma-specific $\mathrm{ICl}$ ) have generated considerable interest as a leading example of novel anticarcinogenic dual immune-virotherapy. Initial results have indicated satisfactory patient treatment safety and moderate improvements in participants' prognoses and disease progression, which is especially important given the high aggressiveness in both melanoma and secondary squamous tumour behaviours. ${ }^{35}$ 


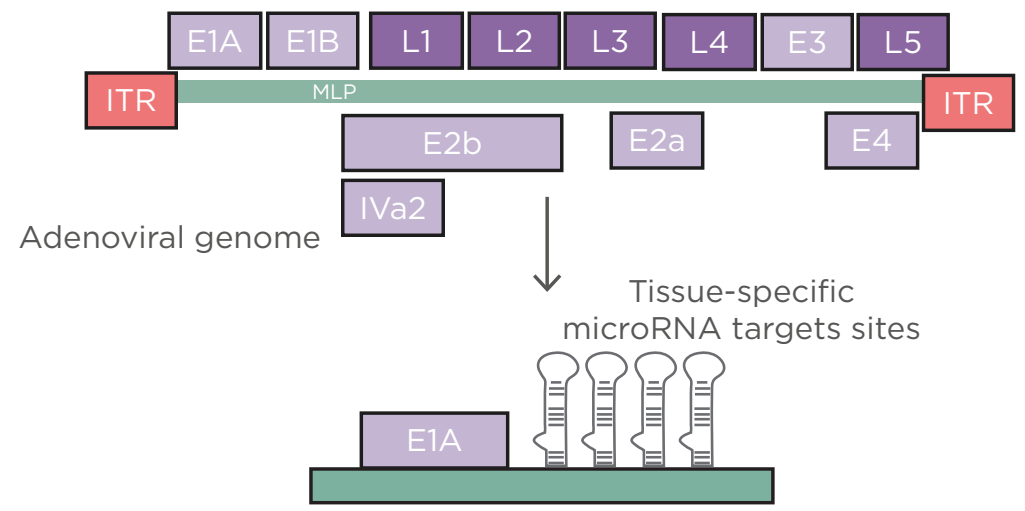

Post-transcriptional

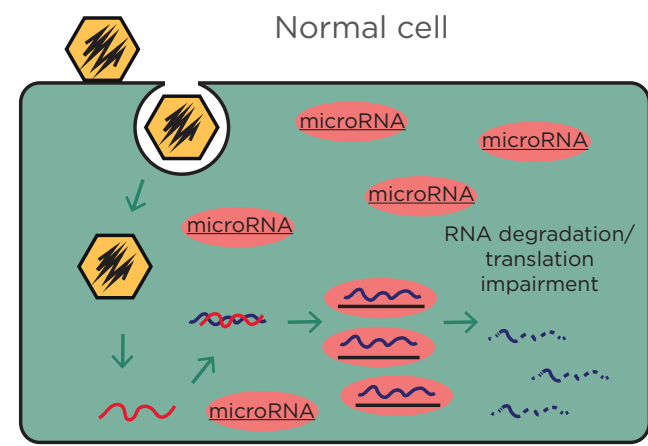

Viral infection but no replication

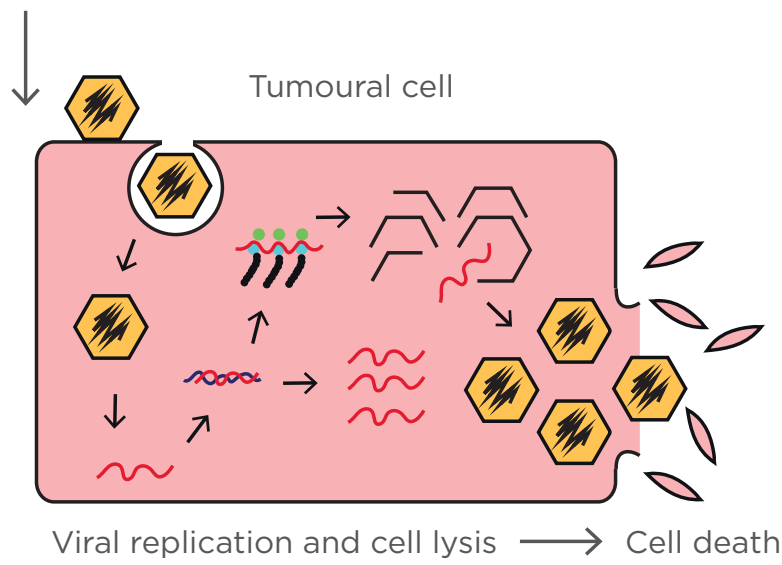

Figure 3: Oncolytic adenovirus controlled by microRNA response element.

Representative depiction of oncolytic adenovirus in vivo function and target cellular subpopulation selectivity, relative to independent and supportive cancer immunotherapy applications. Infected cells may influence both local tumour cell lysis as well as systemic upregulation of immune function and tumour targetability through induced release of pro-immunogenic pro-inflammatory factors.

Adapted from Bofill-De Ros, 2010. ${ }^{52}$

Recent biological methods of tumour antigen expansion have increased the attention for the synthesis and patient personalisation of so-called cancer vaccines, where samples of allogenic inactivated target tumour antigen may be tailored and presented to host immune resources prior to the induction of more standard immunotherapeutic methods.

Extensive modern trials encompassing GVAX (pro-GM-CSF) for pancreatic and prostate cancer ${ }^{56,57}$ demonstrate a significant increase in host recruitment and tumour infiltration of CD8+ T-lymphocytes, with correspondingly significant increases in patient survival and disease state $(p<0.02)$ alongside PD-1 inhibition. ${ }^{58,59}$ Whereas large-scale follow-up remains vital to elucidate applicability of cancer vaccines as a means of augmenting low levels of foundation immune response to cold tumours, ongoing studies strongly suggest such vaccinations may cotransform nonresponsive cold cancer populations towards TIL-inflammation through direct effect while also expanding checkpoint inhibitor functionality through multiplication of relevant special-effector T-lymphocytes. ${ }^{60}$ However, difficulties to practising fully-personalised cancer vaccination alongside immunotherapy endure, which outside of practical implications in resource or time investment is further constrained by advanced disease realities such as patients' deficient T-cell function and incomplete response to initial vaccination. ${ }^{61}$ 


\section{DISCUSSION AND FUTURE DIRECTIONS}

Immunologic intervention is frequently cited as being amongst the most adaptable and promising of treatment options in modern oncology, broadly applicable to the management of a wide array of cancers. However, appropriate knowledge on adaptation and application of constituent methodologies ranging from CAR $T$ cells to oncolytic viruses and novel vaccines remain relatively underdeveloped and an emerging field of inquiry. Further challenged by varying degrees of tumoural immune activity seen within solid malignancies, especially regarding more robust immunomodulatory therapies in non-solid tumour cancers. While the clinical effects and expected adverse events of CAR T therapy to target immunomodulatory tumour antigens such as CD19 alongside adaptability with immune costimulatory domains CD28/CD137 are largely well-characterised in conditions such as diffuse large B-cell lymphoma, ${ }^{62}$ current applications and suitable targets within solid tumours, particularly those challenging immunologically cold malignancies for which CAR T might intuitively prove most beneficial, remains under investigation and its perceived effectiveness still to be well-demonstrated. 63,64

As outlined in this review, ongoing clinical trials on traditionally cold and less immunogenic solid tumours emphasising co-operative combination therapies and immunogenic interactions suggest that translational and personalised cancer immune interventions contain considerable therapeutic value in the realm of solid tumour management. Of further importance is that immunotherapy for solid tumours, unlike haematologic and primary systemic malignancies (i.e., lymphoma), may not exclusively exist as curative in intent. Strong evidence indicate that the gold standard for patient prognosis across many solid tumour cancers is early-stage curative resection, considering the high relapsefree cure rates;"6,66 substantial value may also be derived out of cold tumour immunotherapy as an opportunity for surgery-supportive perioperative care. In illustration, where for nonHodgkin's lymphoma a moderately effective immunotherapeutic response achieved by CAR $T$ infusion may prove only useful for control of disease spread but accompanied by major adverse outcomes with long-term use, the same level of efficaciousness in primary pancreatic cancer could produce previously nonresectable growths (i.e., caused by staging or aggressiveness profile) into the tumour staging range for clinical consideration of operation. ${ }^{67}$ In cold immunologic tumours management, combination approaches may expand the patient and disease profiles associated with treatable characterisation and, through the broadening of operative suitability classification, could provide marked benefits toward epidemiologic cure rates for many cancers.

Against alternative nonsurgical methodologies such as chemo- and radiotherapy for nonsurgical management alone, across limited published studies, immunotherapy has demonstrated slightly reduced systemic side-effect profiles with no significant increases in either patient adverse event frequency or serious adverse event severity. ${ }^{68,69}$ A reasonable expectation would therefore persist in the fact that with greater flexibility and biomarker identification ability within immune-based treatment regimens, more control and minimisation may be exerted on behalf of patients undergoing cancer treatments, which is presently cited amongst the leading instigators for patient cancer therapy nonadherence. ${ }^{70}$ That is not to indicate that long-established anticancer methodologies such as chemo- and radiotherapy no longer have a valuable freestanding niche in the arsenal of cancer treatment options in light of immunotherapeutic medicine progression. Outside of combination therapies to provoke ICD, early systematic treatment of diagnosed malignancies through alternative mechanisms may also reduce the pathologic intratumoural selection of immunologically cold clones less responsive to both physiologic control processes as well as inducible immunotherapy.

Major challenges to generalised adaptation of current immunotherapeutic techniques remain prominent. These encompass the management of patient autoimmunity, side effects through greater modifiable therapeutic selectivity, and a reduction in therapy-associated immunotoxicity. Moving forwards, investigation of cancer immunotherapy's efficacy and tolerability in early-stage disease is of critical importance given the presently limited data. Clinical intuition suggest that existing immunostimulatory modalities likely demonstrate more favourable findings in advanced tumour stages (the 
concurrent focus population of most available trials) given that patient immunocompromise reduces baseline physiologic antitumour defences whilst also limiting host immunotoxicity potential, ${ }^{71-73}$ a major barrier against greater onco-immunology translation. The rise of generalised hyperimmunity (i.e., allergies) and autoimmunity particularly in first-world nations ${ }^{74,75}$ additionally requires clarification upon the future manifestation and clinical role of onco-immunology, specifically in relation to side-effect profile severity and incidence during treatment, upon widespread adaptation.

Pragmatically, continued research and proposed practise of highly individualised precision oncotherapeutics must necessitate scalable and encompassing genetic and immunotherapeutic biomarker repositories. Because of the extreme complexity of tumour microenvironment interactions as well as local-to-global immune relationships, only through accurate and exhaustive bioinformatics databases would bench-to-bedside management guidance prove truly attainable. Likewise, valid reservations remain regarding current practicality and cost-benefit of effectively inducing clinical immunogenicity within less-responsive tumours, ignorant to direct immunotherapeutic intervention. Concerns regarding the practicality and cost-benefit analysis of relatively $\mathrm{ICl}$ dependent modern immunotherapy to cancers with inherently low mutational loads (i.e., forms of pancreatic cancer, which subsequently reduces effectiveness of any $\mathrm{ICl}$ antibodies), physiologic barriers to combination drug therapy penetration (i.e., immune- and chemotherapeutically derived drug entry into the central nervous system through the blood-brain barrier ${ }^{76}$ ), or stillunknown mechanisms for significant observed deviations in patient response given disease status to combination immunotherapy (either baseline or acquired therapy resistance not previously noted $)^{77-79}$ require proactive exploration.

\section{CONCLUSION}

This review of current literature and clinical trials critically analyses and identifies potential current avenues of clinical utility for immunotherapy in the treatment of immunologically cold solid tumour neoplasms. In spite of nascent efficacy data and directly translatable clinical value for immunologically derived approaches independently, it is the position of this paper that combination treatment guidelines incorporating means of immunogenicity induction followed by targeted immunotherapeutic $\mathrm{ICl}$ remain realistic and of critical importance for sustained investigation. As a complement to early detection and surgical resection, immunotherapy demonstrates the exciting concurrent capacity to inform perioperative management of solid tumours as neo-adjuvant care, while also potentially proving curative for malignancies with identifiable and therapeutically targetable markers. This provides a wide scope of application that includes the potential to treat resistant tumours traditionally regarded as being immunologically cold. With the continued identification and functional clarification of further immunologically relevant cellular antigens and receptors as well as tumour microenvironment interrelationships, longestablished boundaries in tumour characterisation alongside associated therapeutic evaluation are increasingly less definite.

A profession-wide shift of healthcare towards personalised medicine and translational therapeutics is now constrained in the realm of immunologically cold solid tumours by a persistent inability to effectively identify and target such neoplasms accurately. Numerous ongoing efforts to clinically induce immunogenicity for consequent immune intervention through chemotherapy, radiotherapy, vector, and vaccine-based modalities are promising but unfinished. Meaningful progress in these fronts will require accompanied advancement in the scientific community's understanding of tumour microenvironment interactions as well as clonal heterogeneity, an ambitious order that will require considerable sustained research. Nonetheless, given the many avenues of potential immunotherapeutic management presently under exploration as well as their apparent untapped clinical potential, eventual introduction of adaptable immunotherapies effective and versatile to both traditionally hot and cold immune activity tumours with therapeutic success still appears a generally reasonable expectation. Combination and novel cancer immunotherapy, by extension personalised medicine of the future, carry great applicability and clinical promise for the efficacious treatment of diverse, challenging malignancy subtypes inclusive of resistant solid tumours exhibiting low immune responsiveness. 


\section{References}

1. Lechner $M$ et al. Chemokines, costimulatory molecules and fusion proteins for the immunotherapy of solid tumors. Immunotherapy. 2011:3(11):1317-40.

2. Couzin-Frankel J. Breakthrough of the year 2013. Cancer immunotherapy. Science. 2013:342(6165):1432-3.

3. Hegasy Illustartions. Cancer Therapy by Inhibition of Negative Immune Regulation (CTLA4, PD1). Available at: https://www.hegasy.de/dwnlds/11 Hegasy CTLA4 PD1 Immunotherapy. pdf. Last accessed: 02 April 2020.

4. Postow $M$ et al. Immune checkpoint blockade in cancer therapy. J Clin Oncol. 2015:33(17):1974-82.

5. Barkholt L, Bregni M. Current immunotherapy for solid tumors. Immunotherapy. 2009;1(3):483-93.

6. de La Cruz-Merino L et al. Breast cancer immunology and immunotherapy: current status and future perspectives. Int Rev Cell Mol Biol. 2017;331:1-53.

7. Disis M. Immunologic biomarkers as correlates of clinical response to cancer immunotherapy. Cancer Immunol Immunother. 2011;60(3):43342.

8. Melero I et al. T-cell and NK-cell infiltration into solid tumors: a key limiting factor for efficacious cancer immunotherapy. Cancer Discov. 2014;4(5):522-6.

9. Galon J et al. Towards the introduction of the 'Immunoscore' in the classification of malignant tumours. J Pathol. 2014;232(2):199209

10. Mlecnik B et al. Integrative analyses of colorectal cancer show Immunoscore is a stronger predictor of patient survival than microsatellite instability. Immunity. 2016;44(3):698-711.

11. Anitei MG et al. Prognostic and predictive values of the Immunoscore in patients with rectal cancer. Clin Cancer Res. 2014;20(7):1891-9.

12. Mlecnik B et al. The tumor microenvironment and Immunoscore are critical determinants of dissemination to distant metastasis. Sci Transl Med. 2016;8(327):327ra26.

13. Pagès $\mathrm{F}$ et al. International validation of the consensus Immunoscore for the classification of colon cancer: a prognostic and accuracy study. Lancet. 2018;391(10135):2128-39.

14. Hermitte F. Biomarkers immune monitoring technology primer: Immunoscore ${ }^{\circledR}$ Colon. J Immunother Cancer. 2016:4:57.

15. Jiang $Y$ et al. ImmunoScore signature: a prognostic and predictive tool in gastric cancer. Ann Surg. 2018:267(3):504-13.

16. Ogino S, Giannakis M.
Immunoscore for (colorectal) cancer precision medicine. Lancet. 2018;391(10135):2084-6.

17. Wargo $\mathrm{J}$ et al. Monitoring immune responses in the tumor microenvironment. Curr Opin mmunol. 2016:41:23-31.

18. Galon J, Bruni D. Approaches to treat immune hot, altered and cold tumours with combination immunotherapies. Nat Rev Drug Discov. 2019;18(3):197-218.

19. Peng $\mathrm{D}$ et al. Epigenetic silencing of TH1-type chemokines shapes tumour immunity and immunotherapy. Nature. 2015:527(7577):249-53.

20. Riley $R$ et al. Delivery technologies fo cancer immunotherapy. Nat Rev Drug Discov. 2019:18(3):175-196.

21. Esteva $\mathrm{F}$ et al. Immunotherapy and targeted therapy combinations in metastatic breast cancer. Lancet Oncol. 2019:20(3):e175-86.

22. Gujar S et al. Heating it up: oncolytic viruses make tumors 'hot' and suitable for checkpoint blockade immunotherapies. Oncolmmunology. 2018;7(8):e1442169.

23. Ciardiello $D$ et al. Immunotherapy of colorectal cancer: challenges for therapeutic efficacy. Cancer Treat Rev. 2019;76:22-32.

24. Sevenich L. Turning 'cold' into 'hot' tumors - opportunities and challenges for radio-immunotherapy against primary and metastatic brain cancers. Front Oncol. 2019;9:163.

25. Menon S et al. Advances in cancer immunotherapy in solid tumors. Cancers. 206;8(12):106.

26. Woo SR et al. STING-dependent cytosolic DNA sensing mediates innate immune recognition of immunogenic tumors. Immunity. 2014:41(5):830-42

27. Novartis Pharmaceuticals. Study of the Safety and Efficacy of MIW815 With PDR001 to Patients With Advanced/Metastatic Solid Tumors or Lymphomas. NCT03172936. https://clinicaltrials.gov/ct2/show/ NCTO3172936.

28. Aduro Biotech, Inc. Safety and Efficacy of MIW815 (ADU-S100) + - Ipilimumab in Patients With Advanced/Metastatic Solid Tumors or Lymphomas. NCTO2675439. https://clinicaltrials.gov/ct2/show/ NCTO2675439.

29. Aduro Biotech, Inc. Efficacy and Safety Trial of ADU-S100 and Pembrolizumab in Head and Neck Cancer. NCT03937141. https:// clinicaltrials.gov/ct2/show/ NCT03937141.

30. Feng $X$ et al. Bioactive modulators targeting STING adaptor in CGASSTING pathway. Drug Discov Today.
2020;25(1):230-7.

31. Helleday T. Making immunotherapy 'cold' tumours 'hot' by chemotherapy-induced mutations - a misconception. Ann Oncol. 2019;30(3):360-1.

32. Sharma P et al. Primary, adaptive, and acquired resistance to cancer immunotherapy. Cell. 2017;168(4):70723.

33. Waldmann T, Morris J. Development of antibodies and chimeric molecules for cancer immunotherapy. Adv Immunol. 2006:90:83-131.

34. Zitvogel $L$ et al. Immune parameters affecting the efficacy of chemotherapeutic regimens. Nat Rev Clin Oncol. 2011;8(3):151-60.

35. Buch S, Chatra L. Immunotherapy and its advances in the management of head-and-neck cancer. CHRISMED J Health Res. 2019;6(4):199-203.

36. Hanson $\mathrm{H}$ et al. Eradication of established tumors by CD8+ T cell adoptive immunotherapy. Immunity. 2000;13(2):265-76.

37. Chiappinelli $\mathrm{K}$ et al. Combining epigenetic and immunotherapy combat cancer. Cancer Res. 2016;76(7):1683-9

38. Yau $\mathrm{H}$ et al. The cancer epigenome: exploiting its vulnerabilities for immunotherapy. Trends Cell Biol. 2019;29(1):31-43.

39. Royal Marsden NHS Foundation Trust. Epigenetic Modulation of the immunE Response in GastrointEstinal Cancers (EMERGE) (EMERGE). NCT03812796. https://clinicaltrials gov/ct2/show/NCT03812796.

40. Cartwright E et al. EMERGE: Epigenetic modulation of the immune response in gastrointestinal cancers. Ann Oncol. 2019:30(5):v251-2.

41. Zheng $Y$ et al. Using chemo-drugs or irradiation to break immune tolerance and facilitate immunotherapy in solid cancer. Cell Immunol. 2015;294(1):549.

42. Sidney Kimmel Comprehensive Cancer Center at Johns Hopkins. A Trial of Boost Vaccinations of Pancreatic Tumor Cell Vaccine. NCT01088789. https://clinicaltrials. gov/ct2/show/NCT01088789.

43. University of Wisconsin, Madison. Phase Ib Study of Stereotactic Body Radiotherapy (SBRT) in Oligometastatic Non-small Lung Cancer (NSCLC) With Dual Immune Checkpoint Inhibition. NCTO3275597. https://clinicaltrials.gov/ct2/show/ NCTO3275597.

44. Vacchelli E et al. Trial watch: chemotherapy with immunogenic cell death inducers. Oncoimmunology. 2012:1(2):179-88.

45. Song A, Lu B. Utility of stereotactic 
ablative radiotherapy/stereotactic body radiation therapy in the setting of oligometastatic non-small cell lung cancer. J Thorac Dis. 2018;10(2):65760 .

46. Nicodemus C. Antibody-based immunotherapy of solid cancers: progress and possibilities. Immunotherapy. 2015;7(8):923-39.

47. Haanen J. Converting cold into hot tumors by combining immunotherapies. Cell. 2017:170(6):1055-6.

48. Ribas A et al. Oncolytic virotherapy promotes intratumoural $\mathrm{T}$ cell infiltration and improves antiPD-1 immunotherapy. Cell. 2017;170(6):1109-19.e10

49. Giraudo L et al. Cytokine-induced killer cells as immunotherapy for solid tumors: current evidence and perspectives. Immunotherapy. 2015;7(9):999-1010.

50. Achard $\mathrm{C}$ et al. Lighting a fire in the tumor microenvironment using oncolytic immunotherapy. EBioMedicine. 2018;31:17-24.

51. Oldford S, Marshall J. Mast cells as targets for immunotherapy of solid tumors. Mol Immunol. 2015;63(1):11324.

52. Bofill-De Ros X. Oncolytic adenovirus controlled by microRNA response element. Available at: https://commons.wikimedia.org/ wiki/File:Oncolytic adenovirus controlled_by_microRNA_response element.png. Last accessed: 02 April 2020.

53. Twumasi-Boateng $\mathrm{K}$ et al. Oncolytic viruses as engineering platforms for combination immunotherapy. Nat Rev Cancer. 2018;18(7):419-32.

54. Amgen. Talimogene Laherparepvec With Pembrolizumab for Recurrent Metastatic Squamous Cell Carcinoma of the Head and Neck (MASTERKEY232 / KEYNOTE-137) (MASTERKEY232). NCTO2626000. https://clinicaltrials.gov/ct2/show/ NCTO2626000.

55. National Cancer Institute (NCl). Talimogene Laherparepvec and Pembrolizumab in Treating Patients With Stage III-IV Melanoma. NCT02965716. https://clinicaltrials. gov/ct2/show/NCTO2965716.

56. Sidney Kimmel Comprehensive
Cancer Center at Johns Hopkins.

A Neoadjuvant Study of Androgen

Ablation Combined With

Cyclophosphamide and GVAX

Vaccine for Localized Prostate

Cancer. NCT01696877. https://

clinicaltrials.gov/ct2/show/ NCT01696877.

57. Sidney Kimmel Comprehensive Cancer Center at Johns Hopkins. Pilot Study With CY, Pembrolizumab, GVAX, and IMC-CS4 (LY3022855) in Patients With Borderline Resectable Adenocarcinoma of the Pancreas. NCT03153410. https://clinicaltrials. gov/ct2/show/NCT03153410.

58. Pandey V, Storz P. Targeting the tumor microenvironment in pancreatic ductal adenocarcinoma. Expert Rev Anticancer Ther. 2019;19(6):473-82

59. Antonarakis E et al. Neoadjuvant randomized trial of degarelix (Deg) \pm cyclophosphamide/GVAX (Cy/ GVAX) in men with high-risk prostate cancer $(\mathrm{PCa}$ ) undergoing radical prostatectomy (RP). J Clin Oncol. 2017;35(Suppl):5077.

60. Schmidt $C$. The benefits of immunotherapy combinations. Nature. 2017;552(7685):S67-9.

61. Srivatsan $\mathrm{S}$ et al. Allogeneic tumor cell vaccines: the promise and limitations in clinical trials. Hum Vaccin Immunother. 2014;10(1):52-63.

62. Lundqvist A, Childs R. Allogeneic hematopoietic cell transplantation as immunotherapy for solid tumors: current status and future directions. $J$ Immunother. 2005;28(4):281-8.

63. Klebanoff $\mathrm{C}$ et al. Prospects for geneengineered $T$ cell immunotherapy for solid cancers. Nat Med. 2016;22(1):2636.

64. Kosti $P$ et al. Perspectives on chimeric antigen receptor $\mathrm{T}$-cell immunotherapy for solid tumors. Front Immunol. 2018:9:1104.

65. Jang JY et al. Actual long-term outcome of extrahepatic bile duct cancer after surgical resection. Ann Surg. 2005;241(1):77-84

66. Simmonds $P$ et al. Surgical resection of hepatic metastases from colorecta cancer: a systematic review of published studies. Br J Cancer. 2006:94(7):982-99.

67. Brooks E et al. Stereotactic ablative radiation therapy combined with immunotherapy for solid tumors. Cancer J. 2016;22(4):257-66.

68. Heaton K, Grimm E. Cytokine combinations in immunotherapy for solid tumors: a review. Cancer Immunol Immunother. 1993;37(4):2139.

69. Bartholeyns $\mathrm{J}$ et al. Adoptive immunotherapy of solid tumors with activated macrophages: experimental and clinical results. Anticancer Res. 1991;11(3):1201-4.

70. Puts $M$ et al. Factors influencing adherence to cancer treatment in older adults with cancer: a systematic review. Ann Oncol. 2014;25(3):564-77.

71. Fessas P et al. Immunotoxicity from checkpoint inhibitor therapy: clinical features and underlying mechanisms. Immunology. 2020;159(2):167-77.

72. Young $A$ et al. The balancing act between cancer immunity and autoimmunity in response to immunotherapy. Cancer Immunol Res. 2018;6(12):1445-52.

73. Vaishnav R et al. Unforeseen consequences of cancer immunotherapy. Int J Nanomed. 2018;3(1). doi: 10.18203/issn.24563994.

74. Osipov A et al. From immune checkpoints to vaccines: The past, present and future of cancer immunotherapy. Adv Cancer Res. 2019;143:63-144.

75. Andersen M. Anti-cancer immunotherapy: breakthroughs and future strategies. Semin Immunopathol. 2019;41(1):1-3

76. Van Tellingen $\mathrm{O}$ et al. Overcoming the blood-brain tumor barrier for effective glioblastoma treatment. Drug Resist Updat. 2015;19:1-12.

77. Mocellin S et al. Adjuvant immunotherapy for solid tumors: from promise to clinical application. Cancer Immunol Immunother. 2002;51(11-12):583-95.

78. Mahoney $\mathrm{K}$ et al. Combination cancer immunotherapy and new immunomodulatory targets. Nat Rev Drug Discov. 2015;14(8):561-584.

79. Pham T et al. An update on immunotherapy for solid tumors: a review. Ann Surg Oncol. 2018;25(11):3404-12. 